\title{
Regularity and uniqueness for a class of solutions to the hydrodynamic flow of nematic liquid crystals
}

\author{
Tao Huang \\ Department of Mathematics, The Pennsylvania State University \\ University Park, PA 16802, USA \\ txh35@psu.edu
}

\begin{abstract}
In this paper, we establish an $\epsilon$-regularity criterion for any weak solution $(u, d)$ to the nematic liquid crystal flow (1.1) such that $(u, \nabla d) \in L_{t}^{p} L_{x}^{q}$ for some $p \geq 2$ and $q \geq n$ satisfying the condition (1.2). As consequences, we prove the interior smoothness of any such a solution when $p>2$ and $q>n$. We also show that uniqueness holds for the class of weak solutions $(u, d)$ the Cauchy problem of the nematic liquid crystal flow (1.1) that satisfy $(u, \nabla d) \in L_{t}^{p} L_{x}^{q}$ for some $p>2$ and $q>n$ satisfying (1.2).
\end{abstract}

\section{Introduction}

For any $n \geq 3$, the hydrodynamic flow of nematic liquid crystals in $\mathbb{R}^{n} \times[0, T]$, for some $0<T<$ $+\infty$, is given by

$$
\begin{cases}u_{t}+u \cdot \nabla u-\Delta u+\nabla P=-\nabla \cdot\left(\nabla d \otimes \nabla d-\frac{1}{2}|\nabla d|^{2} \mathbb{I}_{n}\right) & \text { in } \mathbb{R}^{n} \times(0, T) \\ \nabla \cdot u=0 & \text { in } \mathbb{R}^{n} \times(0, T) \\ d_{t}+u \cdot \nabla d=\Delta d+|\nabla d|^{2} d & \text { in } \mathbb{R}^{n} \times(0, T) \\ (u, d)=\left(u_{0}, d_{0}\right) & \text { on } \mathbb{R}^{n} \times\{0\}\end{cases}
$$

where $u: \mathbb{R}^{n} \times[0, T] \rightarrow \mathbb{R}^{n}$ is the velocity field of underlying incompressible fluid, $d: \mathbb{R}^{n} \times[0, T] \rightarrow S^{2}$ is the director field of nematic liquid crystal molecules, $P: \mathbb{R}^{n} \times[0, T] \rightarrow \mathbb{R}$ is the pressure function, $\nabla \cdot$ denotes the divergence operator on $\mathbb{R}^{n}, \nabla d \otimes \nabla d=\left(\frac{\partial d}{\partial x_{i}} \cdot \frac{\partial d}{\partial x_{j}}\right)_{1<i, j \leq n}$ is the stress tensor induced by the director field $d, \mathbb{I}_{n}$ is the identity matrix of order $n, u_{0}: \mathbb{R}^{n} \rightarrow \mathbb{R}^{n}$ is the initial velocity field with $\nabla \cdot u_{0}=0$, and $d_{0}: \mathbb{R}^{n} \rightarrow S^{2}$ is the initial director field.

The system (1.1) is a simplified version of the Ericksen-Leslie system modeling the hydrodynamics of liquid crystal materials, proposed by Ericksen [2] and Leslie [15] in 1960's. It is a macroscopic continuum description of the time evolution of the material under the influence of both the flow field and the macroscopic description of the microscopic orientation configurations of rod-like liquid crystals. The interested readers can refer to [2], [15], [16], and [18] for more detail. Mathematically, 
the system (1.1) is strongly coupling the Naiver-Stokes equations and the (transported) heat flow of harmonic maps into $S^{2}$.

For $n=2$, Lin-Lin-Wang [17] have proved the existence of global Leray-Hopf type weak solutions to (1.1) with initial and boundary conditions, which is smooth away from finitely many possible singular times (see Hong [6] and Xu-Zhang [23] for related works). Lin-Wang [19] proved the uniqueness for such weak solutions. It remains a very challenge open problem to prove the global existence of Leray-Hopf type weak solutions and partial regularity of suitable weak solutions to (1.1) in higher dimensions. A BKM type blow-up criterion was obtained for the local strong solution to (1.1) for $n=3$ by [9], i.e., if $0<T_{*}<+\infty$ is the maximum time interval of the strong solution to (1.1), then

$$
\int_{0}^{T_{*}}\left(\|\nabla \times u\|_{L^{\infty}}+\|\nabla d\|_{L^{\infty}}^{2}\right) d t=+\infty .
$$

Recently, the local well-posedness of (1.1) was obtained for initial data $\left(u_{0}, d_{0}\right)$ with $\left(u_{0}, \nabla d_{0}\right) \in$ $L_{\text {uloc }}^{3}\left(\mathbb{R}^{3}\right)$, the space of uniformly locally $L^{3}$-integrable functions, of small norm for $n=3$ by [7.

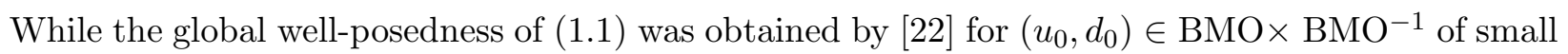
norm for $n \geq 3$.

The existence of global Leray-Hopf weak solutions to the Naiver-Stokes equations has long been established by Leray [14] and Hopf [5]. However the uniqueness (regularity) of Leray-Hopf solutions in dimension three remains largely open. In [20], Serrin proved the so called 'weak-strong' uniqueness, i.e., the uniqueness holds for Leray-Hopf solutions $u, v$ with the same initial data, if $u \in L_{t}^{p} L_{x}^{q}\left(\mathbb{R}^{n} \times[0, T]\right)$, where $p \geq 2$ and $q \geq n$ satisfy

$$
\frac{2}{p}+\frac{n}{q}=1
$$

The smoothness of such solutions was established by Ladyzhenskaya in [13] for $p>2$ and $q>n$. In the fundamental work [3], Escauriaza-Seregin-Šverák have proved the smoothness of Serrin's solutions for the endpoint case $(p, q)=(+\infty, n)$ when $n=3$ (see also [1] for $n \geq 4$ ). Wang 21] proved smoothness of weak solutions $u$ to the heat flow of harmonic maps such that $\nabla u \in$ $L_{t}^{p} L_{x}^{q}\left(\mathbb{R}^{n} \times[0, T]\right)$ with $\frac{2}{p}+\frac{n}{q}=1$ for $n \geq 4$ (or $q \geq 4$ for $2 \leq n<4$, see [11] for the case $2<q<4$ when $2 \leq n<4$ ). In [11], the uniqueness of Serrin's solutions to the heat flow of harmonic maps is also established when $p>2$ and $q>n$. These results motivate us to investigate the regularity and uniqueness of Serrin's $(p, q)$-solutions to the system (1.1) of nematic liquid crystal flows.

Before stating our main theorems, we need to introduce some notations.

Notations: For $1 \leq p, q \leq+\infty, 0<T \leq \infty$, define the Sobolev space

$$
\begin{gathered}
H^{1}\left(\mathbb{R}^{n} \times[0, T], \mathbb{R}^{n}\right)=\left\{f \in L^{2}\left([0, T], H^{1}\left(\mathbb{R}^{n}, \mathbb{R}^{n}\right)\right): \partial_{t} f \in L^{2}\left([0, T], L^{2}\left(\mathbb{R}^{n}, \mathbb{R}^{n}\right)\right)\right\}, \\
\mathbb{E}^{p}\left(\mathbb{R}^{n} \times[0, T], \mathbb{R}^{n}\right)=\left\{f \in L^{p}\left(\mathbb{R}^{n} \times[0, T], \mathbb{R}^{n}\right) \mid \nabla \cdot f=0\right\},
\end{gathered}
$$

and the Morrey space $M^{p, \lambda}(U)$ for $0 \leq \lambda \leq n+2$ and $U=U_{1} \times\left[t_{1}, t_{2}\right] \subset \mathbb{R}^{n} \times \mathbb{R}$ :

$$
M^{p, \lambda}(U)=\left\{f \in L_{\mathrm{loc}}^{p}(U):\|f\|_{M^{p, \lambda}(U)}<+\infty\right\}
$$


where

$$
\begin{gathered}
\|f\|_{M^{p, \lambda}(U)}=\left(\sup _{(x, t) \in U} \sup _{0<r<\min \delta\left((x, t), \partial_{p} U\right)} r^{\lambda-n-2} \int_{P_{r}(x, t)}|f|^{p}\right)^{\frac{1}{p}}, \\
B_{r}(x)=\left\{y \in \mathbb{R}^{n}:|y-x| \leq r\right\}, P_{r}(x, t)=B_{r}(x) \times\left[t-r^{2}, t\right], \partial_{p} U=\left(\partial U_{1} \times\left[t_{1}, t_{2}\right]\right) \cup\left(U_{1} \times\left\{t_{1}\right\}\right),
\end{gathered}
$$

and

$$
\delta\left((x, t), \partial_{p} U\right)=\inf _{(y, s) \in \partial_{p} U} \delta((x, t),(y, s)), \text { and } \delta((x, t),(y, s))=\min \{|x-y|, \sqrt{|t-s|}\} .
$$

Denote $B_{r}$ (or $P_{r}$ ) for $B_{r}(0)$ (or $P_{r}(0)$ respectively). We also recall the weak Morrey space, $M_{*}^{p, \lambda}(U)$, that is the set of functions $f$ on $U$ such that

$$
\|f\|_{M_{*}^{p, \lambda}(U)}^{p}=\sup _{r>0,(x, t) \in U}\left\{r^{\lambda-(n+2)}\|f\|_{L^{p, *}\left(P_{r}(x, t) \cap U\right)}^{p}\right\}<+\infty
$$

where $L^{p, *}\left(P_{r}(x, t) \cap U\right)$ is the weak $L^{p}$-space, that is the collection of functions $v$ on $P_{r}(x, t) \cap U$ such that

$$
\|v\|_{L^{p, *}\left(P_{r}(x, t) \cap U\right)}^{p}=\sup _{a>0}\left\{a^{p}\left|\left\{z \in P_{r}(x, t) \cap U:|v(z)|>a\right\}\right|\right\}<+\infty .
$$

Recall that $(u, d) \in H^{1}\left(\mathbb{R}^{n} \times[0, T], \mathbb{R}^{n} \times S^{2}\right)$ is a weak solution to (1.1) if $(u, d)$ satisfies (1.1) $1^{\text {- }}$ (1.1) 3 in sense of distribution and (1.1) 4 in sense of trace. A weak solution $(u, d) \in H^{1}\left(\mathbb{R}^{n} \times\right.$ $\left.[0, T], \mathbb{R}^{n} \times S^{2}\right)$ of (1.1) if called a Serrin's $(p, q)$-solution, if $(u, \nabla d) \in L_{t}^{p} L_{x}^{q}\left(\mathbb{R}^{n} \times[0, T]\right)$ for some $(p, q)$ satisfying (1.2) . Our first result concerns an $\epsilon_{0}$-regularity criterion for Serrin's $(p, q)$-solutions to (1.1).

Theorem 1.1 There exists $\epsilon_{0}>0$ such that if a weak solution $(u, d) \in H^{1}\left(P_{r}\left(x_{0}, t_{0}\right), \mathbb{R}^{n} \times S^{2}\right)$ to (1.1) satisfies

$$
\|u\|_{L_{t}^{p} L_{x}^{q}\left(P_{r}\left(x_{0}, t_{0}\right)\right)}+\|\nabla d\|_{L_{t}^{p} L_{x}^{q}\left(P_{r}\left(x_{0}, t_{0}\right)\right)} \leq \epsilon_{0}
$$

where $p \geq 2$ and $q \geq n$ satisfy (1.2), then $(u, d) \in C^{\infty}\left(P_{\frac{r}{16}}\left(x_{0}, t_{0}\right)\right)$, and

$$
r\|u\|_{L^{\infty}\left(P_{\frac{r}{16}}\left(x_{0}, t_{0}\right)\right)}+r\|\nabla d\|_{L^{\infty}\left(P_{\frac{r}{16}}\left(x_{0}, t_{0}\right)\right)} \leq C\left(\|u\|_{L_{t}^{p} L_{x}^{q}\left(P_{r}\left(x_{0}, t_{0}\right)\right)}+\|\nabla d\|_{L_{t}^{p} L_{x}^{q}\left(P_{r}\left(x_{0}, t_{0}\right)\right)}\right) .
$$

A direct corollary of Theorem 1.1 is the following regularity theorem for Serrin's $(p, q)$-solutions to (1.1).

Corollary 1.2 For some $0<T<+\infty$, suppose that $(u, d) \in H^{1}\left(\mathbb{R}^{n} \times[0, T], \mathbb{R}^{n} \times S^{2}\right)$ is a weak solution to (1.1) with $(u, \nabla d) \in L_{t}^{p} L_{x}^{q}\left(\mathbb{R}^{n} \times[0, T]\right)$, for some $p>2$ and $q>n$ satisfying (1.2). Then $(u, d) \in C^{\infty}\left(\mathbb{R}^{n} \times(0, T], \mathbb{R}^{n} \times S^{2}\right)$.

Remark 1.3 (i) For the heat flow of harmonic maps and the Navier-Stokes equations, Corollary 1.2 is valid for the end point case $(p, q)=(+\infty, n)$. It is an interesting open question to investigate the regularity of Serrin's solutions to (1.1) in this end point case.

(ii) If $\left(u_{0}, \nabla d_{0}\right) \in L^{\gamma}\left(\mathbb{R}^{n}\right)$ for some $\gamma>n$, then the local existence of Serrin's solutions in $L_{t}^{p} L_{x}^{q}$ for some $p>2$ and $q>n$ can be obtained by the fixed point argument (see e.g., [4] §4). 
As a corollary of Theorem 1.1 and Corollary 1.2 , we can prove the uniqueness of Serrin's $(p, q)$ solutions to (1.1).

Theorem 1.4 For $n \geq 2,0<T<+\infty$, and $i=1,2$, if $\left(u_{i}, d_{i}\right): \mathbb{R}^{n} \times[0, T] \rightarrow \mathbb{R}^{n} \times S^{2}$ are two weak solutions to (1.1) with the same initial data $\left(u_{0}, d_{0}\right): \mathbb{R}^{n} \rightarrow \mathbb{R}^{n} \times S^{2}$. Suppose, in additions, there exists $p>2$ and $q>n$ satisfying (1.2) such that $\left(u_{1}, \nabla d_{1}\right),\left(u_{2}, \nabla d_{2}\right) \in L_{t}^{p} L_{x}^{q}\left(\mathbb{R}^{n} \times[0, T]\right)$. Then $\left(u_{1}, d_{1}\right) \equiv\left(u_{2}, d_{2}\right)$ on $\mathbb{R}^{n} \times[0, T]$.

Remark 1.5 For $n=2$, Lin-Wang [19] have proved the uniqueness of (1.1) for $p=q=4$. More precisely, if, for $i=1,2$,

$$
\left\{\begin{array}{l}
u_{i} \in L^{\infty}\left([0, T], L^{2}\left(\mathbb{R}^{2}, \mathbb{R}^{2}\right)\right) \cap L^{2}\left([0, T], H^{1}\left(\mathbb{R}^{2}, \mathbb{R}^{2}\right)\right) \\
\nabla d_{i} \in L^{\infty}\left([0, T], L^{2}\left(\mathbb{R}^{2}\right)\right) \cap L^{2}\left([0, T], H^{1}\left(\mathbb{R}^{2}\right)\right)
\end{array}\right.
$$

are weak solutions to (1.1) under the same initial condition, then $\left(u_{1}, d_{1}\right) \equiv\left(u_{2}, d_{2}\right)$ on $\mathbb{R}^{2} \times[0, T]$. For $n \geq 3$, Lin-Wang [19] proved the uniqueness, provided that $u_{i} \in C\left([0, T], L^{n}\left(\mathbb{R}^{n}\right)\right)$ and $\nabla d_{i} \in$ $C\left([0, T], L^{n}\left(\mathbb{R}^{n}\right)\right)$ for $i=1,2$.

\section{Proof of Theorem 1.1 and Corollary 1.2}

In this section, we will prove Theorem 1.1 and Corollary 1.2 for nematic liquid crystal flows (1.1). The crucial step is to establish an $\epsilon_{0}$-regularity criterion.

Lemma 2.1 There exists $\epsilon_{0}>0$ such that if $(u, \nabla d) \in L_{t}^{p} L_{x}^{q}\left(P_{1}(0,1)\right)$, for some $p \geq 2$ and $q \geq n$ satisfying (1.2), is a weak solution to (1.1) that satisfies

$$
\|u\|_{L_{t}^{p} L_{x}^{q}\left(P_{1}(0,1)\right)}+\|\nabla d\|_{\left.L_{t}^{p} L_{x}^{q}\left(P_{1}(0,1)\right)\right)} \leq \epsilon_{0}
$$

then $(u, d) \in C^{\infty}\left(P_{\frac{1}{16}}(0,1)\right)$, and

$$
\|u\|_{L^{\infty}\left(P_{\frac{1}{16}}(0,1)\right)}+\|\nabla d\|_{L^{\infty}\left(P_{\frac{1}{16}}(0,1)\right)} \leq C \epsilon_{0} .
$$

Before proving this lemma, we need the following inequality, due to Serrin [20].

Lemma 2.2 For any open set $U \subset \mathbb{R}^{n}$ and any open interval $I \subset \mathbb{R}$, let $f, g, h \in L_{t}^{2} H_{x}^{1}(U \times I)$ and $f \in L_{t}^{p} L_{x}^{q}(U \times I)$ with $3 \leq n \leq q \leq+\infty$ and $2 \leq p \leq+\infty$ satisfying (1.2). Then

$$
\int_{U \times I}|f\|g\| \nabla h| \leq C\|\nabla h\|_{L^{2}(U \times I)}\|g\|_{L_{t}^{2} H_{x}^{1}(U \times I)}^{\frac{n}{q}}\left\{\int_{I}\|f\|_{L^{q}\left(\mathbb{R}^{n}\right)}^{p}\|g\|_{L^{2}\left(\mathbb{R}^{n}\right)}^{2} d t\right\}^{\frac{1}{p}},
$$

where $C>0$ depends only on $n$.

Proof of Lemma 2.1. For any $(x, t) \in P_{\frac{1}{2}}(0,1)$ and $0<r<\frac{1}{2}$, we have, by (2.1),

$$
\|u\|_{L_{t}^{p} L_{x}^{q}\left(P_{r}(x, t)\right)}+\|\nabla d\|_{L_{t}^{p} L_{x}^{q}\left(P_{r}(x, t)\right)} \leq \epsilon_{0} .
$$


We will divide the proof into two claims.

Claim 1. $\nabla d \in L^{\gamma}\left(P_{\frac{1}{2}}(0,1)\right)$ for any $1<\gamma<\infty$, and

$$
\|\nabla d\|_{L^{\gamma}\left(P_{\frac{1}{4}}(0,1)\right)} \leq C(\gamma)\|\nabla d\|_{L_{t}^{p} L_{x}^{q}\left(P_{1}(0,1)\right)} .
$$

To show it, let $d_{1}: P_{r}(x, t) \rightarrow \mathbb{R}^{3}$ solve

$$
\left\{\begin{aligned}
\partial_{t} d_{1}-\Delta d_{1}=0, & \text { in } P_{r}(x, t) \\
d_{1}=d, & \text { on } \partial_{p} P_{r}(x, t) .
\end{aligned}\right.
$$

Set $d_{2}=d-d_{1}$. Multiplying (1.1) 3 and (2.6) by $d_{2}$, subtracting the resulting equations and integrating over $P_{r}(x, t)$, we obtain

$$
\begin{gathered}
\sup _{t-r^{2} \leq \tau \leq t} \int_{B_{r}(x)}\left|d_{2}\right|^{2}(\cdot, \tau)+2 \int_{P_{r}(x, t)}\left|\nabla d_{2}\right|^{2} \\
\leq C \int_{P_{r}(x, t)}\left(|u|\left|d_{2}\right||\nabla d|+|\nabla d|\left|d_{2}\right||\nabla d|\right)=J_{1}+J_{2} .
\end{gathered}
$$

By (2.3), the Poincaré inequality and the Young inequality, we have

$$
\begin{aligned}
\left|J_{1}\right| & \lesssim \begin{cases}\|\nabla d\|_{L^{2}\left(P_{r}(x, t)\right)}\left\|\nabla d_{2}\right\|_{L^{2}\left(P_{r}(x, t)\right)}^{\frac{n}{q}}\left\{\int_{t-r^{2}}^{t}\|u\|_{L^{q}\left(B_{r}(x)\right)}^{p}\left\|d_{2}\right\|_{L^{2}\left(B_{r}(x)\right)}^{2} d \tau\right\}^{\frac{1}{p}}, & p<+\infty \\
\|\nabla d\|_{L^{2}\left(P_{r}(x, t)\right)}\left\|\nabla d_{2}\right\|_{L^{2}\left(P_{r}(x, t)\right)}\|u\|_{L_{t}^{\infty} L_{x}^{n}\left(P_{r}(x, t)\right)}, & p=+\infty,\end{cases} \\
& \leq \begin{cases}\frac{1}{2}\left\|\nabla d_{2}\right\|_{L^{2}\left(P_{r}(x, t)\right)}^{2}+C \epsilon_{0}\|\nabla d\|_{L^{2}\left(P_{r}(x, t)\right)}^{2}+C \epsilon_{0}^{\frac{p}{2}}\left\|d_{2}\right\|_{L_{t}^{\infty} L_{x}^{2}\left(P_{r}(x, t)\right)}^{2}, & p<+\infty \\
\frac{1}{2}\left\|\nabla d_{2}\right\|_{L^{2}\left(P_{r}(x, t)\right)}^{2}+C \epsilon_{0}\|\nabla d\|_{L^{2}\left(P_{r}(x, t)\right)}^{2}, & p=+\infty .\end{cases}
\end{aligned}
$$

Similarly, for $J_{2}$, we have

$$
\left|J_{2}\right| \leq \begin{cases}\frac{1}{2}\left\|\nabla d_{2}\right\|_{L^{2}\left(P_{r}(x, t)\right)}^{2}+C \epsilon_{0}\|\nabla d\|_{L^{2}\left(P_{r}(x, t)\right)}^{2}+C \epsilon_{0}^{\frac{p}{2}}\left\|d_{2}\right\|_{L_{t}^{\infty} L_{x}^{2}\left(P_{r}(x, t)\right)}^{2}, & p<+\infty \\ \frac{1}{2}\left\|\nabla d_{2}\right\|_{L^{2}\left(P_{r}(x, t)\right)}^{2}+C \epsilon_{0}\|\nabla d\|_{L^{2}\left(P_{r}(x, t)\right)}^{2}, & p=+\infty .\end{cases}
$$

Putting these estimates into (2.7), applying (2.4), and choosing sufficiently small $\epsilon_{0}$, we have

$$
\int_{P_{r}(x, t)}\left|\nabla d_{2}\right|^{2} \leq C \epsilon_{0}\|\nabla d\|_{L^{2}\left(P_{r}(x, t)\right)}^{2} .
$$

This, combined with the standard estimate on $d_{1}$, implies that for any $\theta \in(0,1)$,

$$
(\theta r)^{-n} \int_{P_{\theta r}(x, t)}|\nabla d|^{2} \leq C\left(\theta^{2}+\theta^{-n} \epsilon_{0}\right) r^{-n} \int_{P_{r}(x, t)}|\nabla d|^{2} .
$$

By iterations, we obtain for any $(x, t) \in P_{\frac{1}{2}}(0,1), 0<r \leq \frac{1}{2}$ and $0<\alpha<1$,

$$
r^{-n} \int_{P_{r}(x, t)}|\nabla d|^{2} \leq C r^{2 \alpha} \int_{P_{1}(0,1)}|\nabla d|^{2} .
$$

Hence $\nabla d \in \mathcal{M}^{2,2-2 \alpha}\left(P_{\frac{1}{2}}(0,1)\right)$ and

$$
\|\nabla d\|_{\mathcal{M}^{2,2-2 \alpha}\left(P_{\frac{1}{2}}(0,1)\right)} \leq C\|\nabla d\|_{L_{t}^{p} L_{x}^{q}\left(P_{1}(0,1)\right)} .
$$


Now Claim 1 follows by the same estimate of Riesz potentials between parabolic Morrey spaces as in [10] (Theorem 1.5) and [19] (Lemma 2.1).

Claim 2. $u \in L^{\gamma}\left(P_{\frac{1}{4}}(0,1)\right)$ for any $1<\gamma<\infty$, and

$$
\|u\|_{L^{\gamma\left(P_{\frac{1}{4}}(0,1)\right)}} \leq C(\gamma)\|u\|_{L_{t}^{p} L_{x}^{q}\left(P_{1}(0,1)\right)} .
$$

Let $\mathbb{E}^{\gamma}$ be the closure in $L^{\gamma}\left(\mathbb{R}^{n}, \mathbb{R}^{n}\right)$ of all divergence-free vector fields with compact supports. Let $\mathbb{P}: L^{2}\left(\mathbb{R}^{n}, \mathbb{R}^{n}\right) \rightarrow \mathbb{E}^{2}$ be the Leray projection operator. It is well-known that $\mathbb{P}$ can be extended to a bounded linear operator from $L^{\gamma}\left(\mathbb{R}^{n}, \mathbb{R}^{n}\right)$ to $\mathbb{E}^{\gamma}$ for all $1<\gamma<+\infty$. Let $\mathbb{A}=\mathbb{P} \Delta$ denote the Stokes operator.

For any $(x, t) \in P_{\frac{1}{4}}(0,1)$ and $0<r \leq \frac{1}{4}$, let $\eta \in C_{0}^{\infty}\left(P_{2 r}(x, t)\right)$ be such that $0 \leq \eta \leq 1, \eta \equiv 1$ on $P_{r}(x, t),|\nabla \eta| \leq 4 r^{-1}$, and $\left|\partial_{t} \eta\right| \leq 16 r^{-2}$. Let $\left(v, P^{1}\right): \mathbb{R}^{n} \times(0,1) \rightarrow \mathbb{R}^{n} \times \mathbb{R}$ solve

$$
\left\{\begin{aligned}
\partial_{t} v-\Delta v+\nabla P^{1} & =-\nabla \cdot\left(\eta^{2}\left(u \otimes u+\nabla d \otimes \nabla d-\frac{1}{2}|\nabla d|^{2} \mathbb{I}_{n}\right)\right) & & \text { in } \mathbb{R}^{n} \times(0,1) \\
\nabla \cdot v & =0 & & \text { in } \mathbb{R}^{n} \times(0,1) \\
v & =0 & & \text { on } \mathbb{R}^{n} \times\{0\} .
\end{aligned}\right.
$$

Define $w: P_{r}(x, t) \rightarrow \mathbb{R}^{n}$ by $w=u-v$. Then $w$ solves the Stokes equation in $P_{r}(x, t)$ :

$$
\left\{\begin{aligned}
\partial_{t} w-\Delta w+\nabla Q^{1}=0 & \text { in } P_{r}(x, t) \\
\nabla \cdot w=0 & \text { in } P_{r}(x, t) .
\end{aligned}\right.
$$

By the standard theory of linear Stokes' equations, we have that $w \in C^{\infty}\left(P_{r}(x, t)\right)$ and, for any $\theta \in(0,1)$,

$$
\|w\|_{L_{t}^{p} L_{x}^{q}\left(P_{\theta r}(x, t)\right)} \leq C \theta\|w\|_{L_{t}^{p} L_{x}^{q}\left(P_{r}(x, t)\right)} .
$$

To estimate $v$, we apply $\mathbb{P}$ to both sides of the equation (2.13) $)_{1}$ to obtain

$$
\partial_{t} v-\mathbb{A} v=-\mathbb{P} \nabla \cdot\left(\eta^{2}\left(u \otimes u+\nabla d \otimes \nabla d-\frac{1}{2}|\nabla d|^{2} \mathbb{I}_{n}\right)\right) \text { in } \mathbb{R}^{n} \times(0,1) ; v=0 \text { on } \mathbb{R}^{n} \times\{0\} .
$$

By the Duhamel formula, we have

$$
v(t)=-\int_{0}^{t} e^{-(t-\tau) \mathbb{A}} \mathbb{P} \nabla \cdot\left(\eta^{2}\left(u \otimes u+\nabla d \otimes \nabla d-\frac{1}{2}|\nabla d|^{2} \mathbb{I}_{n}\right)\right) d \tau, 0<t \leq 1 .
$$

Now we can apply Fabes-Jones-Riviere [4] Theorem 3.1 (see also Kato [12] page 474, $\left(2.3^{\prime}\right)$ ) to conclude that $v \in L_{t}^{p} L_{x}^{q}\left(\mathbb{R}^{n} \times[0,1]\right)$ and

$$
\begin{aligned}
\|v\|_{L_{t}^{p} L_{x}^{q}\left(\mathbb{R}^{n} \times[0,1]\right)} & \leq C\left(\|\eta u\|_{L_{t}^{p} L_{x}^{q}\left(\mathbb{R}^{n} \times[0,1]\right)}^{2}+\|\eta \nabla d\|_{L_{t}^{p} L_{x}^{q}\left(\mathbb{R}^{n} \times[0,1]\right)}^{2}\right) \\
& \leq C \epsilon_{0}\left(\|u\|_{\left.L_{t}^{p} L_{x}^{q}\left(P_{2 r}(x, t)\right)\right)}+\|\nabla d\|_{L_{t}^{p} L_{x}^{q}\left(P_{2 r}(x, t)\right)}\right) .
\end{aligned}
$$

Putting (2.15) and (2.17) together, we have that for any $\theta \in(0,1)$,

$$
\|u\|_{L_{t}^{p} L_{x}^{q}\left(P_{\theta r}(x, t)\right)} \leq C\left(\theta+\epsilon_{0}\right)\|u\|_{L_{t}^{p} L_{x}^{q}\left(P_{2 r}(x, t)\right)}+C \epsilon_{0}\|\nabla d\|_{L_{t}^{p} L_{x}^{q}\left(P_{2 r}(x, t)\right)}
$$


By Claim 1, we have that for any $\alpha \in(0,1)$, there exists $\epsilon_{0}>0$ depending on $\alpha$ such that

$$
\|\nabla d\|_{L_{t}^{p} L_{x}^{q}\left(P_{2 r}(x, t)\right)} \leq C r^{\alpha}\|\nabla d\|_{L_{t}^{p} L_{x}^{q}\left(P_{1}(0,1)\right)} .
$$

Substituting (2.19) into (2.18) yields

$$
\|u\|_{L_{t}^{p} L_{x}^{q}\left(P_{\theta r}(x, t)\right)} \leq C\left(\theta+\epsilon_{0}\right)\|u\|_{L_{t}^{p} L_{x}^{q}\left(P_{2 r}(x, t)\right)}+C r^{\alpha}\|\nabla d\|_{L_{t}^{p} L_{x}^{q}\left(P_{1}(0,1)\right)} .
$$

It is standard that by choosing $\theta=\theta_{0}(\alpha)>0$ and iterating (2.20) finitely many times, we conclude that for any $(x, t) \in P_{\frac{1}{4}}, 0<r \leq \frac{1}{4}$ and $0<\alpha<1$,

$$
\|u\|_{L_{t}^{p} L_{x}^{q}\left(P_{r}(x, t)\right)} \leq C\left(\|u\|_{L_{t}^{p} L_{x}^{q}\left(P_{1}(0,1)\right)}+\|\nabla d\|_{L_{t}^{p} L_{x}^{q}\left(P_{1}(0,1)\right)}\right) r^{\alpha} .
$$

By Hölder's inequality, (2.21) implies that $u \in \mathcal{M}^{2,2-2 \alpha}\left(P_{\frac{3}{8}}(0,1)\right)$, and

$$
\|u\|_{\mathcal{M}^{2,2-2 \alpha}\left(P_{\frac{3}{8}}(0,1)\right)} \leq C\left[\|u\|_{L_{t}^{p} L_{x}^{q}\left(P_{1}(0,1)\right)}+\|\nabla d\|_{L_{t}^{p} L_{x}^{q}\left(P_{1}(0,1)\right)}\right] .
$$

The higher integrability estimate of $u$ on $P_{\frac{1}{4}}(0,1)$ can be done by the parabolic Riesz potential estimate in parabolic Morrey spaces. Here we will sketch it. Let $\phi \in C_{0}^{\infty}\left(P_{\frac{3}{8}}(0,1)\right)$ such that $0 \leq \phi \leq 1, \phi \equiv 1$ on $P_{\frac{5}{16}}(0,1)$, and

$$
\left|\partial_{t} \phi\right|+|\nabla \phi|+\left|\nabla^{2} \phi\right| \leq C
$$

Define $\widetilde{u}: \mathbb{R}^{n} \times[0,1] \rightarrow \mathbb{R}^{n}$ by

$$
\widetilde{u}(t)=-\int_{0}^{t} e^{-(t-\tau) \mathbb{A}} \mathbb{P} \nabla \cdot\left(\phi^{2}\left(u \otimes u+\nabla d \otimes \nabla d-\frac{1}{2}|\nabla d|^{2} \mathbb{I}_{n}\right)\right) d \tau, 0<t \leq 1 .
$$

Then, as in the proof of Theorem 3.1 (i) of [4], we have that for any $(x, t) \in \mathbb{R}^{n} \times(0,1]$,

$$
|\widetilde{u}(x, t)| \leq C \int_{0}^{t} \int_{\mathbb{R}^{n}} \frac{1}{\delta^{n+1}((x, t),(y, s))}\left(|\phi u|^{2}+|\phi \nabla d|^{2}\right)(y, s) d y d s .
$$

Recall the parabolic Riesz potential of order $1, I_{1}(\cdot)$, is defined by

$$
I_{1}(f)(z):=\int_{\mathbb{R}^{n+1}} \frac{|f(w)|}{\delta^{n+1}(z, w)} d w, f \in L^{1}\left(\mathbb{R}^{n+1}\right) .
$$

Then we have

$$
|\widetilde{u}(x, t)| \leq C I_{1}(F)(x, t), \quad(x, t) \in \mathbb{R}^{n} \times(0,1]
$$

where

$$
F=\phi^{2}\left(|u|^{2}+|\nabla d|^{2}\right) .
$$

By Hölder's inequality, (2.11), and (2.22), we have that $F \in \mathcal{M}^{1,2-2 \alpha}\left(\mathbb{R}^{n+1}\right)$ and

$$
\|F\|_{\mathcal{M}^{1,2-2 \alpha}\left(\mathbb{R}^{n+1}\right)} \leq C\left(\|\nabla d\|_{L_{t}^{p} L_{x}^{q}\left(P_{1}(0,1)\right)}^{2}+\|u\|_{L_{t}^{p} L_{x}^{q}\left(P_{1}(0,1)\right)}^{2}\right) .
$$


Hence, by [10] Theorem 3.1 (ii), we conclude that $\widetilde{u} \in \mathcal{M}_{*}^{\frac{2-2 \alpha}{1-2 \alpha}, 2-2 \alpha}\left(\mathbb{R}^{n} \times[0,1]\right)$, and

$$
\begin{aligned}
\|\widetilde{u}\|_{\mathcal{M}_{*}^{\frac{2-2 \alpha}{1-2 \alpha}, 2-2 \alpha}} & \leq C\|F\|_{\left.\mathbb{R}^{n} \times[0,1]\right)} \\
& \leq C\left(\|\nabla d\|_{L_{t}^{p} L_{x}^{q}\left(P_{1}(0,1)\right)}^{2}+\|u\|_{L_{t}^{p} L_{x}^{q}\left(P_{1}(0,1)\right)}^{2}\right) .
\end{aligned}
$$

As $\lim _{\alpha \uparrow \frac{1}{2}} \frac{2-2 \alpha}{1-2 \alpha}=+\infty$, we have that $\widetilde{u} \in L^{\gamma}\left(P_{\frac{5}{16}}(0,1)\right)$ for any $1<\gamma<+\infty$, and

$$
\|\widetilde{u}\|_{L^{\gamma\left(P_{\frac{5}{16}}\right)}} \leq C(\gamma)\left(\|\nabla d\|_{L_{t}^{p} L_{x}^{q}\left(P_{1}(0,1)\right)}^{2}+\|u\|_{L_{t}^{p} L_{x}^{q}\left(P_{1}(0,1)\right)}^{2}\right) .
$$

Set $\widetilde{w}=u-\widetilde{u}$ on $P_{\frac{5}{16}}(0.1)$. Then it follows from (1.1) and (2.23) that

$$
\partial_{t} \widetilde{w}-\Delta \widetilde{w}+\nabla \widetilde{Q}=0 ; \nabla \cdot \widetilde{w}=0 \text { in } P_{\frac{5}{16}}(0,1) .
$$

By the standard theory of linear Stokes' equations, we have that $\widetilde{w} \in L^{\infty}\left(P_{\frac{1}{4}}(0,1)\right)$, and

$$
\begin{aligned}
\|\widetilde{w}\|_{L^{\infty}\left(P_{\frac{1}{4}}(0,1)\right)} & \leq C\|\widetilde{w}\|_{L^{1}\left(P_{\frac{5}{16}}(0,1)\right)} \leq C\left(\|u\|_{L^{1}\left(P_{\frac{5}{16}}(0,1)\right)}+\|\widetilde{u}\|_{L^{1}\left(P_{\frac{5}{16}}(0,1)\right)}\right) \\
& \leq C\left(\|\nabla d\|_{L_{t}^{p} L_{x}^{q}\left(P_{1}(0,1)\right)}+\|u\|_{L_{t}^{p} L_{x}^{q}\left(P_{1}(0,1)\right)}\right) .
\end{aligned}
$$

It is clear that (2.12) follows from (2.28) and (2.29). This completes the proof of Claim 2 .

Finally, it is not hard to see that by the $W_{\gamma}^{2,1}$-theory for the heat equation and the linear Stokes equation, and the Sobolev embedding theorem, we have that $(u, \nabla d) \in L^{\infty}\left(P_{\frac{1}{8}}(0,1)\right)$. Then the Schauder's theory and the bootstrap argument can imply that $(u, d) \in C^{\infty}\left(P_{\frac{1}{16}}(0,1)\right)$. Furthermore, the estimate (2.2) holds. This completes the proof.

Proof of Corollary 1.2, It is easy to see that when $p>2, q>n$, for any $(x, t) \in \mathbb{R}^{n} \times(0, T]$, we can find $R_{0}>0$ such that

$$
\|u\|_{L_{t}^{p} L_{x}^{q}\left(P_{R_{0}}(x, t)\right)}+\|\nabla d\|_{L_{t}^{p} L_{x}^{q}\left(P_{R_{0}}(x, t)\right)} \leq \epsilon_{0},
$$

where $\epsilon_{0}$ is given in Lemma 2.1. By Theorem 1.1, we conclude that $(u, d) \in C^{\infty}\left(P_{\frac{R_{0}}{16}}(x, t)\right)$. This completes the proof of Theorem 1.2

\section{Proof of Theorem 1.4}

In this section, we will prove Theorem 1.4. To do this, we need the following estimate.

Lemma 3.1 For $T>0$, suppose that $(u, d)$ is a weak solution to (1.1) in $\mathbb{R}^{n} \times(0, T]$, which satisfies the assumption of Theorem 1.4. Then $(u, d) \in C^{\infty}\left(\mathbb{R}^{n} \times(0, T], \mathbb{R}^{n} \times S^{2}\right)$, and there exists $t_{0}>0$ such that for $0<t \leq t_{0}$, it holds

$$
\sup _{0<\tau \leq t} \sqrt{\tau}\left(\|u(\tau)\|_{L^{\infty}\left(\mathbb{R}^{n}\right)}+\|\nabla d(\tau)\|_{L^{\infty}\left(\mathbb{R}^{n}\right)}\right) \leq C\left(\|u\|_{L_{t}^{p} L_{x}^{q}\left(\mathbb{R}^{n} \times[0, t]\right)}+\|\nabla d\|_{L_{t}^{p} L_{x}^{q}\left(\mathbb{R}^{n} \times[0, t]\right)}\right) .
$$

In particular, we have

$$
\lim _{t \downarrow 0^{+}} \sqrt{t}\left(\|u\|_{L^{\infty}\left(\mathbb{R}^{n}\right)}+\|\nabla d\|_{L^{\infty}\left(\mathbb{R}^{n}\right)}\right)=0 .
$$


Proof. Let $\epsilon_{0}$ be given by Lemma 2.1. Since $p>2$ and $q>n$ satisfy (1.2), for any $0<\epsilon \leq \epsilon_{0}$ we can find $t_{0}>0$ such that for any $0<\tau \leq \sqrt{t_{0}}$

$$
\|u\|_{L_{t}^{p} L_{x}^{q}\left(\mathbb{R}^{n} \times\left[0, \tau^{2}\right]\right)}+\|\nabla d\|_{L_{t}^{p} L_{x}^{q}\left(\mathbb{R}^{n} \times\left[0, \tau^{2}\right]\right)} \leq \epsilon .
$$

For any $x_{0} \in \mathbb{R}^{n}$, define

$$
\begin{aligned}
& \bar{u}(y, s)=\tau u\left(x_{0}+y \tau, s \tau^{2}\right) \\
& \bar{P}(y, s)=\tau^{2} P\left(x_{0}+y \tau, s \tau^{2}\right) \\
& \bar{d}(y, s)=d\left(x_{0}+y \tau, s \tau^{2}\right) .
\end{aligned}
$$

Then $(\bar{u}, \bar{P}, \bar{d})$ is a weak solution to (1.1) on $P_{1}(0,1)$, and by (3.3),

$$
\|\bar{u}\|_{L_{t}^{p} L_{x}^{q}\left(P_{1}(0,1)\right)}+\|\nabla \bar{d}\|_{L_{t}^{p} L_{x}^{q}\left(P_{1}(0,1)\right)} \leq \epsilon .
$$

By Lemma 2.1, we conclude that

$$
|\bar{u}(0,1)|+|\nabla \bar{d}(0,1)| \leq C\left(\|\bar{u}\|_{L_{t}^{p} L_{x}^{q}\left(P_{1}(0,1)\right)}+\|\nabla \bar{d}\|_{L_{t}^{p} L_{x}^{q}\left(P_{1}(0,1)\right)}\right) .
$$

By rescaling, this implies

$$
\tau\left(\left|u\left(x_{0}, \tau^{2}\right)\right|+\left|\nabla d\left(x_{0}, \tau^{2}\right)\right|\right) \leq C\left(\|u\|_{L_{t}^{p} L_{x}^{q}\left(\mathbb{R}^{n} \times\left[0, \tau^{2}\right]\right)}+\|\nabla d\|_{L_{t}^{p} L_{x}^{q}\left(\mathbb{R}^{n} \times\left[0, \tau^{2}\right]\right)}\right) \leq C \epsilon .
$$

Taking supremum over all $x_{0} \in \mathbb{R}^{n}$ completes the proof.

Proof of Theorem 1.4: By (3.2), we have that for any $\epsilon>0$, there exists $t_{0}=t_{0}(\epsilon)>0$ such that

$$
\begin{aligned}
\mathcal{A}\left(t_{0}\right)= & \sum_{i=1}^{2}\left[\sup _{0 \leq t \leq t_{0}} \sqrt{t}\left(\left\|u_{i}(t)\right\|_{L^{\infty}\left(\mathbb{R}^{n}\right)}+\left\|\nabla d_{i}(t)\right\|_{L^{\infty}\left(\mathbb{R}^{n}\right)}\right)\right. \\
& \left.+\left(\left\|u_{i}\right\|_{\left.L_{t}^{p} L_{x}^{q}\left(\mathbb{R}^{n} \times\left[0, t_{0}\right]\right)\right)}+\left\|\nabla d_{i}\right\|_{\left.L_{t}^{p} L_{x}^{q}\left(\mathbb{R}^{n} \times\left[0, t_{0}\right]\right)\right)}\right)\right] \leq \epsilon .
\end{aligned}
$$

It suffices to show $\left(u_{1}, d_{1}\right)=\left(u_{2}, d_{2}\right)$ on $\mathbb{R}^{n} \times\left[0, t_{0}\right]$. To do so, let $u=u_{1}-u_{2}$ and $d=d_{1}-d_{2}$. Applying $\mathbb{P}$ to both $(1.1)_{1}$ for $u_{1}$ and $u_{2}$ and taking the difference of resulting equations, we have that

$$
\left\{\begin{array}{l}
u_{t}-\mathbb{A} u=-\mathbb{P} \nabla \cdot\left(u \otimes u_{1}+u_{2} \otimes u+\nabla d \otimes \nabla d_{1}+\nabla d_{2} \otimes \nabla d+\left(\left|\nabla d_{1}\right|+\left|\nabla d_{2}\right|\right)|\nabla d| \mathbb{I}_{n}\right), \\
\nabla \cdot u=0 \\
d_{t}-\Delta d=\left[\left(\nabla d_{1}+\nabla d_{2}\right) \cdot \nabla d d_{2}+\left|\nabla d_{1}\right|^{2} d\right]-\left[u \cdot \nabla d_{1}+u_{2} \cdot \nabla d\right], \\
\left.(u, d)\right|_{t=0}=(0,0) .
\end{array}\right.
$$

By the Duhamel formula, we have that for any $0<t \leq t_{0}$,

$$
u(t)=-\int_{0}^{t} e^{-(t-\tau) \mathbb{A}} \mathbb{P} \nabla \cdot\left(u \otimes u_{1}+u_{2} \otimes u+\nabla d \otimes \nabla d_{1}+\nabla d_{2} \otimes \nabla d+\left(\left|\nabla d_{1}\right|+\left|\nabla d_{2}\right|\right)|\nabla d| \mathbb{I}_{n}\right) d \tau,
$$




$$
d(t)=\int_{0}^{t} e^{-(t-\tau) \Delta}\left(\left(\nabla d_{1}+\nabla d_{2}\right) \cdot \nabla d d_{2}+\left|\nabla d_{1}\right|^{2} d-u \cdot \nabla d_{1}-u_{2} \cdot \nabla d\right) d \tau .
$$

For $0<t \leq t_{0}$, set

$$
\Phi(t)=\|u\|_{\left.L_{t}^{p} L_{x}^{q}\left(\mathbb{R}^{n} \times[0, t]\right)\right)}+\|\nabla d\|_{\left.L_{t}^{p} L_{x}^{q}\left(\mathbb{R}^{n} \times[0, t]\right)\right)}+\sup _{0 \leq \tau \leq t}\|d(\cdot, \tau)\|_{L^{\infty}\left(\mathbb{R}^{n}\right)} .
$$

By (3.9) and the standard estimate on the heat kernel, we obtain that

$$
\begin{aligned}
\|\nabla d(t)\|_{L^{q}\left(\mathbb{R}^{n}\right)} \leq & C\left[\sum_{i=1}^{2} \int_{0}^{t}(t-\tau)^{\frac{1}{p}-1}\left\|\nabla d_{i}\right\|_{L^{q}\left(\mathbb{R}^{n}\right)}\|\nabla d\|_{L^{q}\left(\mathbb{R}^{n}\right)} d \tau\right. \\
& +\|d\|_{L^{\infty}\left(\mathbb{R}^{n}\right)} \int_{0}^{t}(t-\tau)^{\frac{1}{p}-1}\left\|\nabla d_{1}\right\|_{L^{q}\left(\mathbb{R}^{n}\right)}^{2} d \tau \\
& +\int_{0}^{t}(t-\tau)^{\frac{1}{p}-1}\left\|\nabla d_{1}\right\|_{L^{q}\left(\mathbb{R}^{n}\right)}\|u\|_{L^{q}\left(\mathbb{R}^{n}\right)} d \tau \\
& \left.+\int_{0}^{t}(t-\tau)^{\frac{1}{p}-1}\left\|u_{2}\right\|_{L^{q}\left(\mathbb{R}^{n}\right)}\|\nabla d\|_{L^{q}\left(\mathbb{R}^{n}\right)} d \tau\right] .
\end{aligned}
$$

By the standard Riesz potential estimate in $L^{p}$-spaces (see [4] Theorem 3.0), we see that $\nabla d \in$ $L_{t}^{p} L_{x}^{q}\left(\mathbb{R}^{n} \times\left[0, t_{0}\right]\right)$, and

$$
\begin{aligned}
\|\nabla d\|_{L_{t}^{p} L_{x}^{q}\left(\mathbb{R}^{n} \times\left[0, t_{0}\right]\right)} \leq & C\left[\sum_{i=1}^{2}\left\|\nabla d_{i}\right\|_{\left.L_{t}^{p} L_{x}^{q}\left(\mathbb{R}^{n} \times\left[0, t_{0}\right]\right)\right)}\|\nabla d\|_{L_{t}^{p} L_{x}^{q}\left(\mathbb{R}^{n} \times\left[0, t_{0}\right]\right)}\right. \\
& +\|d\|_{L^{\infty}\left(\mathbb{R}^{n} \times\left[0, t_{0}\right]\right)}\left\|\nabla d_{1}\right\|_{L_{t}^{p} L_{x}^{q}\left(\mathbb{R}^{n} \times\left[0, t_{0}\right]\right)}^{2} \\
& +\left\|\nabla d_{1}\right\|_{L_{t}^{p} L_{x}^{q}\left(\mathbb{R}^{n} \times\left[0, t_{0}\right]\right)}\|u\|_{L_{t}^{p} L_{x}^{q}\left(\mathbb{R}^{n} \times\left[0, t_{0}\right]\right)} \\
& \left.+\left\|u_{2}\right\|_{L_{t}^{p} L_{x}^{q}\left(\mathbb{R}^{n} \times\left[0, t_{0}\right]\right)}\|\nabla d\|_{L_{t}^{p} L_{x}^{q}\left(\mathbb{R}^{n} \times\left[0, t_{0}\right]\right)}\right] \\
\leq & C \mathcal{A}\left(t_{0}\right) \Phi\left(t_{0}\right) .
\end{aligned}
$$

Similarly, by using the estimate of Theorem 3.1 (i) of [4], we have that $u \in L_{t}^{p} L_{x}^{q}\left(\mathbb{R}^{n} \times\left[0, t_{0}\right]\right)$, and

$$
\|u\|_{L_{t}^{p} L_{x}^{q}\left(\mathbb{R}^{n} \times\left[0, t_{0}\right]\right)} \leq C \mathcal{A}\left(t_{0}\right) \Phi\left(t_{0}\right) .
$$

Now we need to estimate $\sup _{0 \leq \tau \leq t_{0}}\|d(\cdot, \tau)\|_{L^{\infty}\left(\mathbb{R}^{n}\right)}$. We claim

$$
\|d\|_{L^{\infty}\left(\mathbb{R}^{n} \times\left[0, t_{0}\right]\right)} \leq C \mathcal{A}\left(t_{0}\right) \Phi\left(t_{0}\right) .
$$

To show (3.13), let $H(x, t)$ be the heat kernel of $\mathbb{R}^{n}$. By (3.9), we have

$$
\begin{aligned}
|d(x, t)|=\mid & \int_{0}^{t} \int_{\mathbb{R}^{n}} H(x-y, t-\tau)\left(\left(\nabla d_{1}+\nabla d_{2}\right) \cdot \nabla d d_{2}+\left|\nabla d_{1}\right|^{2} d\right)(y, \tau) d y d \tau \\
& -\int_{0}^{t} \int_{\mathbb{R}^{n}} H(x-y, t-\tau)\left(u \cdot \nabla d_{1}+u_{2} \cdot \nabla d\right)(y, \tau) d y d \tau \mid \\
\leq C & {\left[\int_{0}^{t} \int_{\mathbb{R}^{n}} H(x-y, t-\tau) K(y, \tau) d y d \tau\right.} \\
& \left.+\int_{0}^{t} \int_{\mathbb{R}^{n}} H(x-y, t-\tau)\left|\nabla d_{1}\right|^{2}(y, \tau) d y d \tau \cdot \sup _{0 \leq \tau \leq t}\|d(\cdot, \tau)\|_{L^{\infty}\left(\mathbb{R}^{n}\right)}\right],
\end{aligned}
$$


where

$$
K(y, \tau):=\sum_{i=1}^{2}\left(\left|u_{i}\right|+\left|\nabla d_{i}\right|\right)(|u|+|\nabla d|)(y, \tau) .
$$

By (3.7), we have that for any $0<t \leq t_{0}$,

$$
\begin{aligned}
& \int_{0}^{t} \int_{\mathbb{R}^{n}} H(x-y, t-\tau) K(y, \tau) d y d \tau \\
\leq & \mathcal{A}\left(t_{0}\right) \int_{0}^{t}(t-\tau)^{-\frac{n}{2}} \tau^{-\frac{1}{2}} \int_{\mathbb{R}^{n}}(|u|+|\nabla d|) \exp \left(-\frac{|x-y|^{2}}{4(t-\tau)}\right) d y d \tau \\
\leq & \mathcal{A}\left(t_{0}\right)\left\|(t-\tau)^{-\frac{n}{2 q}} \tau^{-\frac{1}{2}}\right\|_{L^{\frac{p}{p-1}([0, t])}}\||u|+|\nabla d|\|_{L_{t}^{p} L_{x}^{q}\left(\mathbb{R}^{n} \times[0, t]\right)} \\
\leq & C \mathcal{A}\left(t_{0}\right) \Phi\left(t_{0}\right),
\end{aligned}
$$

where we have used Hölder inequality and

$$
\begin{aligned}
\left\|(t-\tau)^{-\frac{n}{2 q}} \tau^{-\frac{1}{2}}\right\|_{L^{\frac{p}{p-1}}([0, t])}^{\frac{p}{p-1}} & =t^{\left(\frac{1}{2}-\left(\frac{n}{2 q}+\frac{1}{p}\right)\right) \frac{p}{p-1}} \int_{0}^{1}(1-\tau)^{-\frac{n p}{2(p-1) q}} \tau^{-\frac{p}{2(p-1)}} d \tau \\
& =\int_{0}^{1}(1-\tau)^{-\frac{p-2}{2(p-1)}} \tau^{-\frac{p}{2(p-1)}} d \tau<+\infty
\end{aligned}
$$

as (i) $\frac{n}{2 q}+\frac{1}{p}=\frac{1}{2}$, and (ii) $2<p<+\infty$ yields $\frac{p}{2(p-1)}<1$ and $\frac{p-2}{2(p-1)}<1$.

Similarly, we can obtain that for $0 \leq t \leq t_{0}$,

$$
\int_{0}^{t} \int_{\mathbb{R}^{n}} H(x-y, t-\tau)\left|\nabla d_{1}\right|^{2}(y, \tau) d y d \tau \leq C \mathcal{A}^{2}\left(t_{0}\right)
$$

Putting (3.15) and (3.16) into (3.14) and taking supremum over $(x, t) \in \mathbb{R}^{n} \times\left[0, t_{0}\right]$, we have

$$
\sup _{0 \leq t \leq t_{0}}\|d\|_{L^{\infty}\left(\mathbb{R}^{n}\right)} \leq C \mathcal{A}\left(t_{0}\right) \Phi\left(t_{0}\right)+C \mathcal{A}^{2}\left(t_{0}\right) \sup _{0 \leq t \leq t_{0}}\|d\|_{L^{\infty}\left(\mathbb{R}^{n}\right)}
$$

Therefore, if we choose $\epsilon \leq \sqrt{\frac{1}{2 C}}$ so that $C \mathcal{A}^{2}\left(t_{0}\right) \leq C \epsilon^{2} \leq \frac{1}{2}$, then we obtain (3.13).

Putting (3.11), (3.12) and (3.13) together, and choosing $\epsilon \leq \frac{1}{2 C}$, we obtain

$$
\Phi\left(t_{0}\right) \leq C \mathcal{A}\left(t_{0}\right) \Phi\left(t_{0}\right) \leq \frac{1}{2} \Phi\left(t_{0}\right) .
$$

This implies that $\Phi\left(t_{0}\right)=0$ and hence $\left(u_{1}, d_{1}\right) \equiv\left(u_{2}, d_{2}\right)$ on $\mathbb{R}^{n} \times\left[0, t_{0}\right]$. If $t_{0}<T$, then we can repeat the argument for $t \in\left[t_{0}, T\right]$ and eventually show that $\left(u_{1}, d_{1}\right) \equiv\left(u_{2}, d_{2}\right)$ on $\mathbb{R}^{n} \times[0, T]$. This completes the proof.

Acknowledgements. The paper is part of my Ph.D. thesis in University of Kentucky. I would like to thank my advisor Professor Changyou Wang for his helpful discussion and constant encouragement. 


\section{References}

[1] H. J. Dong, D. P. Du, The Navier-Stokes equations in the critical Lebesgue space. Comm. Math. Phys. 292 (2009), no. 3, 811-827.

[2] J. L. Ericksen, Hydrostatic theory of liquid crystal. Arch. Rational Mech. Anal., 9 (1962), 371-378.

[3] L. Escauriaza, G. Seregin, V. Šverák, $L^{3, \infty}$-solutions of Navier-Stokes equations and backward uniqueness (In Russian). Usp. Mat. Nauk 58 (2003), 3-44; translation in Russ. Math. Surv. 58 (2003), 211-250.

[4] E. Fabes, F. Jones, N. Riviere, The initial value problem for the Navier-Stokes equations with date in $L^{p}$. Arch. Rational Mech. Anal., 45 (1972), 222-240.

[5] E. Hopf, Über die Anfangswertaufgabe für die hydrodynamischen Grundgleichungen. Math. Nachrichten, 4 (1950-51), 213-231.

[6] M. C. Hong, Global existence of solutions of the simplified Ericksen-Leslie system in dimension two. Calc. Var. \& PDE, 40 (2011), no. 1-2, 15-36.

[7] J. Hineman, C. Y. Wang, Well-posedness of nematic liquid crystal flow in $L_{\text {uloc }}^{3}\left(\mathbb{R}^{3}\right)$. arXiv: 1208.5965 .

[8] T. Huang, Regularity and uniqueness of some geometric heat flows and its applications. 2013, Ph.D. Dissertation: http://uknowledge.uky.edu/math_etds/10.

[9] T. Huang, C. Y. Wang, Blow up criterion for nematic liquid crystal flows. Comm. Partial Differential Equations, 37 (2012), 875-884,

[10] T. Huang, C. Y. Wang, Notes on the regularity of harmonic map systems. Proc. Amer. Math. Soc., 138 (6) (2010), 2015-2023.

[11] T. Huang, C. Y. Wang, On uniqueness of heat flow of harmonic maps. Preprint, arXiv: 1208.1470 .

[12] T. Kato, Strong $L^{p}$-solutions of the Navier-Stokes equation in $\mathbb{R}^{m}$, with applications to weak solutions. Math. Z., 187 (1984), 471-480.

[13] O. A. Ladyzhenskaya, On uniqueness and smoothness of generalized solutions to the NaviverStokes equations. Zap. Nauchn. Sem. Leningrad. Otdel. Mat. Inst. Steklov. (LOMI) 5 (1967), 169-185; English transl., Sem. Math. V.A. Steklov Math. Inst. Leningrad 5 (1969), 60-66.

[14] J. Leray, Sur le mouvement d'un liquide visqueux emplissant l'espace. Acta Math., 63 (1934), 193-248. 
[15] F. M. Leslie, Some constitutive equations for liquid crystals. Arch. Rational Mech. Anal., 28 (1968), 265-283.

[16] F. H. Lin, Nonlinear theory of defects in nematic liquid crystal: phase transition and flow phenomena. Comm. Pure Appl. Math., 42 (1989), 789-814.

[17] F. H. Lin, J. Y. Lin, C. Y. Wang, Liquid crystal flows in two dimensions. Arch. Ration. Mech. Anal. 197 (2010), no. 1, 297-336.

[18] F. H. Lin, C. Liu, Nonparabolic Dissipative Systems Modeling the Flow of Liquid Crystals. Comm. Pure. Appl. Math., Vol. XLVIII, (1995), 501-537.

[19] F. H. Lin, C. Y. Wang, On the uniqueness of heat flow of harmonic maps and hydrodynamic flow of nematic liquid crystals. Chin. Ann. Math., 31B (6) (2010), 921-938.

[20] J. Serrin, The initial value problem for the Navier-Stokes equations. In Nonlinear Problems (Proc. Sympos., Madison, Wis., pages 69-98. Univ. of Wisconsin Press, Madison, Wis., 1963).

[21] C. Y. Wang, Heat flow of harmonic maps whose gradients belong to $L_{x}^{n} L_{t}^{\infty}$. Arch. Rational Mech. Anal., 188 (2008), 309-349.

[22] C. Y. Wang, Well-posedness for the heat flow of harmonic maps and the liquid crystal flow with rough initial data. Arch. Ration. Mech. Anal. 2000 (2011), no. 1, 1-19.

[23] X. Xu, Z. F. Zhang, Global regularity and uniqueness of weak solution for the 2-D liquid crystal flows. J. Diff. Eqns., 256 (2012), 1169-1181. 